\title{
Stabilité politique, qualité des institutions et tourisme en Afrique
}

Political Stability, Institutional Quality and Tourism in Africa

Torcia-Chanelle Banengaï-Koyama, Patrice Ongono et Trésor Ekom

\section{OpenEdition}

\section{Journals}

\section{Édition électronique}

URL : https://journals.openedition.org/tourisme/3683

DOI : $10.4000 /$ tourisme.3683

ISSN : 2492-7503

Éditeur

Association Mondes du tourisme

\section{Référence électronique}

Torcia-Chanelle Banengaï-Koyama, Patrice Ongono et Trésor Ekom, «Stabilité politique, qualité des institutions et tourisme en Afrique », Mondes du Tourisme [En ligne], 19 | 2021, mis en ligne le 15 septembre 2021, consulté le 17 septembre 2021. URL : http://journals.openedition.org/tourisme/3683 ; DOI : https://doi.org/10.4000/tourisme.3683

Ce document a été généré automatiquement le 17 septembre 2021.

\section{c)}

Mondes du tourisme est mis à disposition selon les termes de la licence Creative Commons Attribution - Pas d'Utilisation Commerciale - Pas de Modification 4.0 International. 


\title{
Stabilité politique, qualité des institutions et tourisme en Afrique
}

\author{
Political Stability, Institutional Quality and Tourism in Africa \\ Torcia-Chanelle Banengaï-Koyama, Patrice Ongono et Trésor Ekom
}

\section{Introduction}

Le secteur touristique a le mérite de stimuler, d'une part, la croissance économique en créant des emplois tout en luttant contre la pauvreté et, d'autre part, de protéger les patrimoines culturels et naturels tout en promouvant l'autonomisation des communautés locales (UNCTAD, 2017). Le tourisme est devenu un passeport pour la prospérité et une force de transformation pour améliorer des millions de vies. Chaque année, près de 1,2 milliard de personnes voyagent à travers le monde. Chaque jour, plus de 3 millions de personnes franchissent des frontières internationales (UNWTO, 2017). Pour réaliser le programme de développement durable à l'horizon 2030, les pays doivent exploiter la puissance du tourisme (UNWTO, 2017). Avec plus d'un milliard de touristes internationaux par an à travers le monde, le tourisme est devenu un secteur économique de premier plan. Sa contribution au produit intérieur brut (PIB) mondial s'élève à $10 \%$ et il contribue à hauteur de $6 \%$ aux exportations mondiales en 2015. De nombreux pays émergents et en développement (le Burkina Faso, la Gambie, Haïti, Madagascar, le Rwanda et la Tanzanie) ont fait du tourisme leur première activité d'exportations. Au total, en 2013, le tourisme a généré 924 milliards de dollars US et s'est classé en quatrième position en termes d'exportations dans le monde après les secteurs des ressources naturelles, des produits chimiques et des produits alimentaires. En 2014 et 2019, le tourisme s'est classé en troisième position devant les produits alimentaires (UNWTO, 2013, 2019). Ces chiffres mettent en lumière le potentiel du tourisme à relever certains défis contemporains, notamment ceux de la croissance, du développement inclusif et de la préservation de l'environnement, même si l'histoire montre que les pays ne peuvent pas compter sur le tourisme comme seul moyen de sortir de la pauvreté ou pour parvenir au développement durable. Construit autour de 
millions de rencontres interculturelles, le tourisme permet également de mieux comprendre le monde au-delà de nos frontières, première étape de la construction de la paix dans/entre les communautés et les nations (UNWTO, 2015).

2 L'année 2017 a été désignée comme l'année du tourisme durable. En même temps, elle reste l'année record en termes de nombre de touristes internationaux, avec 1323 millions de touristes (en hausse de 84 millions par rapport à 2016) dont $50 \%$ en Europe, $25 \%$ en Asie, $16 \%$ en Amérique et $5 \%$ en Afrique (UNWTO, 2017, 2016b). La contribution du tourisme à la croissance, à l'emploi et au commerce en Afrique s'est accrue depuis 1960. Les arrivées de touristes internationaux en Afrique augmentent en moyenne de $6 \%$ et $5 \%$ par an entre 1995 et 2014 et entre 2015 et 2017 respectivement. Suivant la même tendance, les recettes d'exportations du tourisme augmentent en moyenne de $9 \%$ par an sur la même période. La contribution moyenne du tourisme au PIB dans les pays africains est passée de 69 milliards de dollars US entre 1995 et 1998 à 166 milliards de dollars US entre 2011 et 2014, soit de 6,8 \% à 8,5 \% du PIB. En outre, le tourisme a généré plus de 21 millions d'emplois en moyenne sur la période 2011-2014, représentant 7,1\% des emplois en Afrique. L'industrie du tourisme a soutenu 1 emploi sur 14 en Afrique et 1 emploi sur 11 dans le monde entre 2011 et 2014 (UNWTO, 2014).

3 Les défis actuels majeurs auxquels les pays africains font face sur le plan économique sont la diversification, la transformation structurelle et la croissance inclusive. Sur le plan politique, ces défis comprennent la stabilité politique, le renforcement de la qualité des institutions et la sécurité. S'agissant du plan économique, le tourisme est un secteur florissant avec de grandes potentialités, susceptibles de répondre à ces défis. C'est un secteur complexe qui regroupe des activités interdépendantes et indissociables tant tangibles (i.e., réseaux de transport, hébergement, restauration, visites guidées et souvenirs, ainsi que des services connexes, tels les services bancaires, d'assurance et de sécurité) qu'intangibles (i.e., la relaxation, la culture, l'évasion, l'aventure et les différentes expériences). Le tourisme permet donc de promouvoir la diversification et la transformation structurelle de l'économie à travers les liens intersectoriels. Le tourisme reste en outre un service à forte intensité de main-d'œuvre. Les employés du secteur touristique sont constitués pour 60 à $70 \%$ de femmes et pour $50 \%$ de jeunes âgés de 25 ans et plus, c'est-à-dire les groupes généralement marginalisés dans les autres secteurs (UNCTAD, 2017). Ces chiffres montrent l'efficacité du tourisme en tant qu'instrument de lutte contre la pauvreté et contre l'inégalité sociale en incluant les groupes les plus vulnérables sur le marché du travail. Le tourisme constitue donc un levier de croissance avec de nombreux effets multiplicateurs.

Cependant, sur le plan politique, de nombreux facteurs entravent le développement du tourisme. Comme tout produit d'exportations, le tourisme est très sensible à l'environnement politique, plus encore lorsqu'il s'agit de la sécurité des touristes. Ces derniers sont parfois contraints de changer de destination ou d'annuler leur voyage. Or, les pays ayant souffert de violences voient le nombre d'arrivées de touristes décliner. L'Afrique souffre malheureusement de son image en tant que destination touristique. Elle est souvent décrite comme une destination à risque, sujette à des évènements violents, des maladies, des conflits inter et intraétatiques, une instabilité politique, des troubles sociaux, des services de police inadéquats et des mesures de sécurité limitées. Les menaces et attaques terroristes exacerbent cette situation dans certaines régions touristiques comme au Mali, à Djibouti, en Tunisie, en Somalie, au Kenya et en Ouganda (Yap et Saha, 2013 ; Neumayer, 2004). 
5 La stabilité politique et la qualité des institutions font partie des premiers critères de sélection pour le choix d'une destination touristique. Aussi merveilleuse que puisse être une destination, lorsque cette dernière est instable, elle est directement substituable. Les pays qui ont fait des efforts de stabilité ces dernières années rendent leur destination plus attrayante (Saha et al., 2017 ; Das et Dirienzo, 2009, 2010).

Un grand nombre d'études économiques suggèrent que la stabilité politique (Asongu et al., 2019; UNCTAD, 2017 ; Elshaer et Saad, 2017 ; Pratt et Liu, 2017 ; Causevic et Lynch, 2011 ; Llorca-Vivero, 2008) et la qualité des institutions (Kazaryanze et al., 2018; Kim et al., 2018; Saha et Yap, 2015; Yap et Saha, 2013; Su et Lin, 2014) sont vitales pour le développement du tourisme. Ces auteurs démontrent que les pays stables avec une assez bonne qualité des institutions sont les plus visités. Cependant, l'effet de l'interaction entre la stabilité politique et la qualité des institutions sur le tourisme reste très peu étudié dans la littérature actuelle. Aussi, cet article a pour ambition de combler cette lacune.

7 La présente étude vise à analyser l'effet de l'interaction entre la stabilité politique et la qualité des institutions sur le tourisme dans les pays africains. De ce fait, nous étudions d'abord le lien entre la stabilité politique et le tourisme, puis le lien entre la qualité des institutions et le tourisme. Enfin, l'effet de l'interaction entre la stabilité politique et la qualité des institutions sur le tourisme est mis en évidence. Au regard de la littérature, la première contribution majeure de cet article est l'analyse de l'effet de l'interaction entre stabilité politique et la qualité des institutions sur le tourisme en Afrique. La deuxième est l'utilisation de plusieurs mesures (individuelles et composites) de la stabilité politique et de la qualité des institutions pour attester de la robustesse des résultats en s'appuyant sur les bases de données du Worldwide Governance Indicators (WGI) et de l'International Country Risk Guide (ICRG). Plus précisément, ces indicateurs sont évalués individuellement afin d'analyser leurs effets respectifs et un effet global est testé à travers un indice composite.

8 La suite de l'article est structurée de la manière suivante : la section 1 présente une revue de littérature des effets de l'interaction entre la stabilité politique et la qualité des institutions sur le tourisme; la section 2 présente les données et la stratégie économétrique de démonstration; la section 3 présente les résultats; la section 4 fait des recommandations, avant la conclusion.

\section{Revue de littérature}

9 L'analyse de la relation entre l'environnement politique et le tourisme est couverte par une littérature abondante depuis la contribution séminale de Matthews (1974). Néanmoins, la majorité des travaux se focalise sur l'instabilité politique, les conflits politiques et les guerres civiles (Gozgor et Ongon, 2017 ; Ivanov et al., 2017 ; Pratt et Liu, 2016 ; Sönmez, 1998 ; Llorca-Vivero, 2008 ; Ingram et al., 2013 ; Alvarez et Campo, 2014 ; Saha et Yap, 2014) et le terrorisme (Asongu et al., 2019a ; Liu et Pratt, 2017 ; Neumayer et Plümper, 2016, 2009; Plümper et Neumayer, 2010; Feridum, 2011; Asongu et Nwachukwu, 2017b).

10 Selon l'étude de Hall et O'Sullivan (1996), la stabilité politique est déterminante pour le développement d'une économie en général et pour le tourisme en particulier. En effet, l'instabilité politique et la sécurité sont des conditions préalables à une mobilité 
touristique. Il est donc important de bien comprendre les contours du concept de stabilité politique.

11 Dans cet article, la stabilité politique se définit comme la combinaison de paix négative et de paix positive, selon les termes du Global Peace Index (IEP, 2019). La paix négative se définit comme l'absence de violence ou de peur de violence. La paix positive se construit autour des attitudes, des institutions et des structures qui créent et soutiennent des sociétés pacifiques. La paix positive peut également être utilisée pour mesurer la résilience d'une société face aux chocs sans pour autant retomber dans les conflits. La paix positive et la paix négative sont liées. L'existence de paix positive accroît la probabilité que les conflits se terminent rapidement et sans violence postérieure. La résolution des conflits libère des ressources pour la poursuite de politiques de croissance et de développement, qui à leur tour, conduisent à des niveaux plus élevés de paix positive et à un cercle vertueux de développement pacifique.

\subsection{Stabilité politique et tourisme}

12 Le tourisme constitue une importante source de développement économique pour de nombreux pays non industrialisés. En effet, la contribution des recettes touristiques à la formation du PIB ne cesse de croître pour de nombreux pays d'Afrique subsaharienne $^{1}$ (OMT, 2017 ; Adu-Ampong et al., 2020). Cette hausse a pour effet de réduire la dépendance à l'égard des recettes des matières premières. Les services touristiques sont également sources d'emploi, aussi bien pour la main d'œuvre qualifiée que peu qualifiée (Levantis et Gani, 2000). Malheureusement, ces pays sont le théâtre de nombreuses violences.

13 Les voyageurs modernes se préoccupent prioritairement de leur sécurité et sont sensibles aux violences sociales (Nyaruwata et al., 2013). L'absence de stabilité politique dans une région conduit les touristes à opter pour des destinations plus stables, ou tout simplement à renoncer au voyage (Chingarande, 2014 ; Hall et O'Sullivan, 1996). Les touristes ont le désir de se détendre sans souci (Nyaruwata et al., 2013). Lancaster (1971) écrit que les touristes consomment une combinaison de certaines caractéristiques d'une destination plutôt qu'un seul bien. Aussi, un pays dont les principales attractions sont le climat et les plages est très vulnérable aux scènes de violence politique car d'autres destinations offriront un large choix de substitution aux touristes (Neumayer, 2004). Richter et Waugh (1986) soulignent que le tourisme est souvent l'une des premières victimes des guerres, révolutions ou conflits. Même si les sites touristiques sont sécurisés, l'attraction touristique peut décliner précipitamment lorsque les conditions politiques semblent incertaines. De plus, même lorsque les caractéristiques d'un pays sont très appréciées et difficilement substituables, les attaques contre les touristes peuvent nuire considérablement à l'industrie touristique. À titre illustratif, les attentats de ces dernières années en France ont réduit considérablement le nombre de touristes internationaux, à l'instar des printemps arabes en Égypte, Tunisie, Algérie, Syrie, etc., du 11 septembre aux États-Unis, de la présence de Boko-Haram au Nigeria et dans le nord du Cameroun (UNCTAD, 2017 ; UNWTO, 2016b).

14 Par ailleurs, l'instabilité politique dans un pays peut avoir des externalités sur des pays frontaliers ou qui lui sont connectés par leur commerce international (UNCTAD, 2017 ; Neumayer et Plümper, 2016; Enders et Sandler, 1991; Enders et al., 1992). Cette situation peut être la conséquence du couplage des destinations touristiques. Par 
exemple, le tourisme aux Maldives et à Zanzibar peut être affecté par la violence au Sri Lanka et au Kenya, car les Maldives et Zanzibar sont des destinations populaires pour les voyageurs provenant du Sri Lanka et du Kenya, respectivement. Dans la même logique, Neumayer (2004) suggère que les pays voisins d'un spot touristique bénéficient d'un effet de substitution de destination tant qu'ils ne sont pas eux-mêmes perçus comme directement affectés par les événements de violence.

En outre, l'instabilité politique, même de courte durée, peut avoir un impact considérable et continu en donnant une image négative de la destination touristique. Dans ce cas de figure, l'activité touristique ne peut reprendre que si la destination retrouve une image de stabilité politique. Tout dépend de la durée de l'événement violent et de sa couverture médiatique. Les pays qui présentent une image négative en raison d'épisodes passés de violence tentent d'ailleurs souvent d'améliorer leur image par des campagnes publicitaires agressives (Sönmez et al., 1999; Enders et Sandler, 1991 ; Enders et al., 1992 ; UNCTAD, 2017).

16 Les faits de violence peuvent avoir des conséquences directes et indirectes sur le secteur touristique. Directement, l'instabilité politique conduit, à court terme, à une diminution du nombre de touristes, voire à une baisse des recettes. À long terme, les recettes touristiques peuvent diminuer encore plus fortement que le nombre de touristes à mesure que la nature des profils touristiques évolue ; par exemple, lorsque les touristes plus riches et plus sensibles à la sécurité sont supplantés par des touristes plus sensibles aux prix et preneurs de risques. De manière indirecte, le signalement des conflits et de l'instabilité politique a une incidence sur les décisions d'investissement et de marketing des destinations; ce qui se traduit par une baisse des activités due à une réduction des réservations et initie un cercle vicieux. En effet, les troubles politiques entraînent une baisse de la demande touristique et un déclin de l'investissement privé. Cette situation est la conséquence d'une perte de confiance et d'investissements publics en baisse étant donné que les problèmes de sécurité redéfinissent les priorités d'allocation des ressources. Dans la même veine, les infrastructures touristiques sont susceptibles d'être détruites lors des guerres, des attaques terroristes, des émeutes et des manifestations violentes. Outre les infrastructures, les patrimoines culturels et naturels classés aux patrimoines mondiaux de l'UNESCO peuvent également être détruits, avec une irréversibilité des coûts de restauration.

De nombreuses études empiriques montrent non seulement que l'instabilité politique et le terrorisme ont des impacts négatifs sur le tourisme, mais encore que l'instabilité politique a des impacts plus graves sur le tourisme que les attaques terroristes (Neumayer, 2004 ; Yap et Saha, 2013 ; Asongu et al., 2019). La littérature concernant la relation entre la stabilité politique et le tourisme suggère une double causalité entre la stabilité politique et le tourisme allant de la stabilité politique au tourisme (LlorcaVivero, 2008; Elshaer et Saad, 2017; UNCTAD, 2017) et du tourisme à la stabilité (Causevic et Lynch, 2011 ; UNCTAD, 2017 ; Pratt et Liu, 2017).

\subsection{Qualité des institutions et tourisme}

Les études antérieures traitant du lien entre les institutions et le tourisme étaient axées sur le développement et la croissance du secteur touristique, ainsi que sur la gestion de leurs conséquences environnementales et sociales (Adu-Ampong, 2019; Adu-Ampong et al., 2020). Dans la grande majorité de la littérature, les institutions sont perçues comme 
des sources de contraintes et de législateurs de pouvoir (Kim et al., 2018; Adu-Ampong, 2019). Nunkoo et al. (2012) et Adu-Ampong (2017) montrent que la confiance dans les institutions du tourisme est importante pour la bonne gouvernance du secteur, mais la qualité institutionnelle générale importe également. Mosdale (2014) soutient également cette idée. Das et Dirienzo (2010) sont les premiers à conduire une étude générale des effets de la qualité des institutions sur le tourisme. Ils analysent économiquement les effets de la corruption sur le tourisme et montrent une relation négative entre la corruption et le tourisme. En outre, en analysant les effets de l'instabilité politique, du terrorisme et de la corruption sur les arrivées des touristes dans des sites patrimoines mondiaux de l'UNESCO, Yap et Saha (2013) trouvent une corrélation négative entre la corruption et les arrivées des touristes.

Les institutions auxquelles nous faisons allusion ici sont définies, par Burki et Perry (1998, p. 11), comme « un ensemble de règles formelles et informelles ${ }^{2}$ qui régissent les actions des individus et des organisations dans une société ». Les institutions jouent un rôle fondamental dans le développement économique. Elles permettent: (i) une bonne visibilité de l'avenir en le rendant moins incertain, (ii) une réduction des coûts de transaction et (iii) une bonne coordination entre les structures et les interactions humaines (Mantzavinos, 2001 ; Scott, 1995).

Diverses raisons conduisent à penser que la qualité des institutions influence la demande de tourisme international. D'abord, le tourisme est assimilé aux services échangeables et, en tant que tel, il est soumis aux coûts de transaction engendrés par des externalités lors de la production et de la consommation des services touristiques. La baisse des coûts de transaction liée à l'offre de tourisme international dans une destination peut modifier la courbe de l'offre, au point que le pays en vienne à satisfaire une demande effective de tourisme plus grande pour tous les niveaux de prix (Kim et al., 2018). Les coûts de transaction sont générés lors de tout échange, tant par la recherche d'informations et la négociation que lors de la prise de décision ou l'exécution (Dahlman, 1979; Williamson, 1979). La complexité des transactions touristiques fait intervenir plusieurs participants, ce qui renforce la segmentation de marché et donc augmente les coûts de transaction (North et Thomas, 1973; Palmer et Bejou, 1995 ; Van Niekerk, 2014). Le bon développement du tourisme nécessite des institutions fortes et stables susceptibles de garantir le maintien de l'ordre et d'établir un mécanisme efficace pour résoudre les litiges liés à l'expérience du consommateur et pour protéger les droits de propriété des fournisseurs de services.

De nombreux facteurs de production touristique ont un degré de spécificité élevée, si bien qu'investir dans ces facteurs exige d'avoir confiance dans la protection des droits de propriété et des garanties contractuelles des investisseurs (Kim et al., 2018). Chaque destination est souvent unique dans la combinaison entre environnement naturel et contexte historique et culturel. Cette spécificité montre l'inélasticité de certains facteurs de production clés par rapport à l'offre (Eilat et Einva, 2004; Sinclair et Stabler, 1997). Plus la spécificité d'un actif développé dans la transaction collaborative est élevée, plus le risque d'investir dans cet actif est important, car cela entraîne des coûts de liquidation très élevés. En l'absence d'une forte assurance sur la protection des droits de propriété par le système juridique et dans la mise en œuvre effective des contrats, les investissements domestiques et étrangers dans les actifs touristiques seront probablement faibles (Kim et al., 2018 ; Williamson, 1979). 

de transaction. En effet, les capacités de gouvernance et d'administration efficaces et transparentes déterminent dans une large mesure la compétitivité d'une destination (Saha et Yap, 2015 ; Das et Dirienzo, 2010). Ainsi, un pays ne doit pas seulement mettre en place des politiques touristiques attrayantes, il doit également faciliter l'émergence d'institutions stables, équitables et créatives, qui doivent être largement acceptées sur le plan social.

En résumé, la qualité des institutions est positivement associée aux demandes internationales de tourisme en raison de la réduction des coûts de transaction, de la cohérence, de la crédibilité et de la transparence qu'elle permet. En outre, l'impartialité et l'efficacité du système judiciaire permet une bonne résolution des conflits, tant entre fournisseurs qu'entre fournisseurs et clients (Kim et al., 2018; Tang, 2018). Cet effet de la qualité des institutions va au-delà des frontières en impactant également le tourisme régional (Restrepo et Clavé, 2019).

\section{Méthodologie}

24 Cette section présente : (i) les variables à utiliser, (ii) la spécification économétrique, (iii) les résultats des estimations et (iv) le traitement du biais d'endogénéité.

\subsection{Construction des différentes variables}

25 L'objectif principal de cet article est d'évaluer l'effet de l'interaction entre la stabilité politique et la qualité des institutions sur la demande de tourisme international en Afrique sur la période 1996-2017. Plusieurs techniques économétriques sont utilisées à cette fin. D'abord, le panel non dynamique (effets fixes ou aléatoires) selon l'orientation des tests de spécification de Fisher, du multiplicateur de Lagrange et de Hausman. Ensuite, le panel dynamique à l'aide de l'estimateur de la méthode des moments généralisés (generalized method of moments, GMM) en différence d'Arellano et Bond (1991) en vue de prendre en compte le dynamisme d'une destination touristique et de contrôler le potentiel biais d'endogénéité.

Les études ayant analysé les effets de la stabilité politique et de la qualité des institutions sur la demande de tourisme sur un grand échantillon sont très limitées, notamment pour l'Afrique. Très peu utilisent en outre les techniques économétriques de notre article, à l'exception de Neumayer (2004) qui a utilisé le panel non dynamique et dynamique pour déterminer l'impact de la violence politique sur le tourisme. Par rapport aux études précédentes, cette étude est la première qui évalue l'effet de l'interaction entre la stabilité politique et la qualité des institutions sur le tourisme.

Le présent article se distingue des travaux de Neumayer (2004) et de Yap et Saha (2013) par la zone géographique étudiée, les données et le procédé utilisés. Les données sur la stabilité politique et la qualité des institutions (variables indépendantes d'intérêt) proviennent des bases du Worldwide Governance Indicators (WGI) et de l'International Country Risk Guide (ICRG). Les données du WGI serviront à tester la robustesse des résultats. La stabilité politique est mesurée par les indices de stabilité politique de la Banque mondiale, d'absence de violence/terrorisme du WGI et de conflit interne (IntConf) et externe (ExtTens), de tensions religieuses (ReliTens) et ethniques (Ethtens) de 
l'ICRG. La qualité des institutions est mesurée par les indices de qualité de la bureaucratie, de stabilité du gouvernement (GovStab), de corruption (Corrup), de démocratie (DemoAcc) et de règle et loi (LawOrd) de l'ICRG et par les cinq autres indices $\mathrm{du}$ WGI. Pour chacune des variables ayant plusieurs indicateurs, un indice composite est construit en utilisant le logarithme de la somme ( $L n S P$ et LnQI) de tous les indicateurs pour les variables de l'ICRG. En ce qui concerne les indicateurs provenant du WGI, une somme simple est faite, elle varie dorénavant entre $-7,5$ et 7,5. Les indices du WGI sont rangés de la plus faible qualité $(-2,5)$ à la plus haute qualité $(2,5)$. Nous attendons un effet positif de la stabilité politique, de la qualité des institutions et de leur interaction sur le tourisme.

La demande effective de tourisme, variable dépendante, est mesurée par le nombre effectif d'arrivées de touristes internationaux (InITD) ou par les recettes touristiques (lnITR). La variable dépendante principale est le nombre d'arrivées de touristes. Ce choix est déterminé par le fait qu'il est plus facile et précis de compter le nombre de touristes que d'évaluer les dépenses des touristes au cours de leurs séjours. Toutefois, les recettes touristiques serviront de mesure alternative. Ces données sont fournies par la base des Indicateurs de développement dans le monde (World Development Indicators, WDI) de la Banque mondiale.

Les autres variables indépendantes, dites de contrôle, sont : (i) le produit intérieur brut par tête (lnGDP), variable proxy du niveau de revenu que le touriste pourrait allouer à son séjour ; (ii) la parité de pouvoir d'achat (lnIPC), mesurée par le taux de change réel ou l'indice des prix à la consommation; et (iii) le degré de similarité des pays, mesuré par la variable proxy population urbaine (lnUP). Des signes positifs sont attendus pour toutes les variables. Toutes les variables sont calculées en valeurs logarithmiques pour minimiser le risque d'hétéroscédasticité. La description complète des variables est présentée à l'annexe 3. La matrice de corrélation des indicateurs de la stabilité politique et de la qualité des institutions est également présentée à l'annexe 2.

Tableau 1. Statistiques descriptives

\begin{tabular}{|l|l|l|l|l|l|}
\hline & $\mathbf{( 1 )}$ & $\mathbf{( 2 )}$ & $\mathbf{( 3 )}$ & $\mathbf{( 4 )}$ & $\mathbf{( 5 )}$ \\
\hline Variables & $\mathbf{N}$ & Moyennes & Écarts-types & Min. & Max. \\
\hline LnIPC & 682 & 1.885 & 0.363 & -1.527 & 2.532 \\
\hline LnGDP & 704 & 2.989 & 0.481 & 2.011 & 4.158 \\
\hline LnITD & 704 & 5.608 & 0.722 & 3.699 & 7.148 \\
\hline LnITR & 704 & 8.285 & 0.815 & 5 & 10.13 \\
\hline LnUP & 704 & 6.719 & 0.508 & 5.587 & 7.976 \\
\hline BurQua & 704 & 1.359 & 0.769 & 0 & 3.500 \\
\hline Corrup & 704 & 2.156 & 0.838 & 0 & 5 \\
\hline GovtStab & 704 & 8.496 & 1.788 & 3 & 12 \\
\hline
\end{tabular}




\begin{tabular}{|l|l|l|l|l|l|}
\hline LawOrd & 704 & 3.147 & 1.093 & 0.500 & 6 \\
\hline DemoAcc & 704 & 3.144 & 1.171 & 0 & 5.500 \\
\hline EthTens & 704 & 3.546 & 1.152 & 0 & 6 \\
\hline ExtConf & 704 & 9.880 & 1.415 & 3 & 12 \\
\hline IntConf & 704 & 8.473 & 1.783 & 1 & 12 \\
\hline RelTens & 704 & 4.221 & 1.304 & 0 & 6 \\
\hline SP & 704 & 28.80 & 5.291 & 10 & 41 \\
\hline QI & 704 & 18.30 & 3.149 & 8 & 29.50 \\
\hline LnSP & 704 & 1.451 & 0.0854 & 1 & 1.613 \\
\hline LnQI & 704 & 1.256 & 0.0787 & 0.903 & 1.470 \\
\hline
\end{tabular}

\subsection{Formalisation du modèle économétrique}

Cet article évalue l'effet de l'interaction entre la stabilité politique et la qualité des institutions sur la demande effective de tourisme international sur un panel de 32 pays africains de 1996 à 2017 (cf. annexe 1 pour la liste des pays). Cet effet est analysé à l'aide d'une spécification en panel. Les données de panel présentent l'avantage de fournir plus d'informations et de considérer certaines caractéristiques individuelles et temporelles non prises en compte en série temporelle ou en coupe transversale. Par ailleurs, elles présentent moins de colinéarité, plus de degré de liberté et plus d'efficience en estimation (Baltagi, 2008). À l'issue des tests de spécification de Fisher ${ }^{3}$, du multiplicateur de Lagrange ${ }^{4}$ et de Hausman ${ }^{5}$, le modèle à effets aléatoires est retenu. En effet, les résultats des tests de spécification montrent une absence d'effets fixes dans l'échantillon retenu, ce qui conduit au choix du modèle à effets aléatoires.

Nous estimons la relation entre la stabilité politique, la qualité des institutions et la demande de tourisme en utilisant les modèles suivants :

$\operatorname{lnITD}$ ou $\operatorname{lnITR} R^{6}=f(\operatorname{LnSP}, \operatorname{Ln} Q I, \ln \mathrm{DDP}, \operatorname{lnIPC}, \ln U P)(1)$

$\operatorname{lnITD} D_{i t}=\alpha_{0}+\alpha_{i} \operatorname{LnSP}+\beta_{i} \operatorname{LnQI} I_{i t}+\left(\mu_{i t}+\varepsilon_{i t}\right)(2)$

où $i$ est l'indice pays, $t$ l'indice temporel,

ITD : la demande effective de tourisme international,

ITR : les recettes touristiques internationales,

$S P$ : la matrice des variables représentant la stabilité politique,

QI : la matrice des variables représentant la qualité des institutions,

$\alpha$ et $\beta$ sont les matrices de coefficients estimés.

Dans un premier temps, l'estimation des effets de la stabilité politique sur le tourisme est faite sans variables de contrôle en se basant sur le modèle (3). Ensuite, les variables de contrôle sont ajoutées (4) pour tenir compte des autres facteurs qui influencent la demande touristique. 
$\operatorname{lnITD_{it}}=\alpha_{0}+\alpha_{i} \operatorname{LnSP} P_{i t}+\left(\mu_{i t}+\varepsilon_{i t}\right)(3)$

$\operatorname{lnITD} D_{i t}=\alpha_{0}+\alpha_{i} \operatorname{LnSP} P_{i t}+\gamma_{i} X_{i t}+\left(\mu_{i t}+\varepsilon_{i t}\right)(4)$

où $X$ est la matrice des variables de contrôle.

Dans un deuxième temps, la relation entre la qualité des institutions et le tourisme est évaluée comme précédemment sans variables de contrôle (5) et avec variables de contrôle (6).

$\operatorname{lnITD} D_{i t}=\alpha_{0}+\beta_{i} \operatorname{Ln} Q I_{i t}+\left(\mu_{i t}+\varepsilon_{i t}\right)(5)$

$\operatorname{lnITD} D_{i t}=\alpha_{0}+\beta_{i} \operatorname{LnQI} I_{i t}+Y_{i} X_{i t}+\left(\mu_{i t}+\varepsilon_{i t}\right)(6)$

Enfin, l'estimation de l'équation (7) dans laquelle nous analysons l'effet de l'interaction entre la stabilité politique et la qualité des institutions (notée ) sur le tourisme avec variables de contrôle.

$\operatorname{lnITD} D_{i t}=\alpha_{0}+\beta_{i} \ln Q I \times \ln S P_{i t}+\gamma_{i} X_{i t}+\left(\mu_{i t}+\varepsilon_{i t}\right)(7)$

\subsection{Résultats d'estimation}

Cette section analyse les résultats des estimations des effets de la stabilité politique, de la qualité des institutions et de l'interaction entre la stabilité politique et la qualité des institutions sur la demande effective de tourisme international. Le tableau 2 présente les résultats des estimations à partir du modèle à effets aléatoires entre la stabilité politique et la demande effective de tourisme international ${ }^{7}$.

Tableau 2. Effets de la stabilité politique sur le tourisme (modèle à effets aléatoires)

\begin{tabular}{|l|l|l|l|l|}
\hline & $(\mathbf{1})$ & $(\mathbf{2})$ & $(\mathbf{5})$ & $\mathbf{( 6 )}$ \\
\hline Variables & LnITD & LnITD & LnITR & LnITR \\
\hline & & & & \\
\hline EthTens & $-0.0649^{* * *}$ & $-0.0343^{* *}$ & $-0.0863^{* * *}$ & $-0.0602^{* * *}$ \\
\hline & $(0.0210)$ & $(0.0138)$ & $(0.0308)$ & $(0.0230)$ \\
\hline ExtConf & $-0.0657^{* * *}$ & $-0.00434^{* *}$ & -0.0263 & $-0.0376^{*}$ \\
\hline & $(0.0153)$ & $(0.0103)$ & $(0.0225)$ & $(0.0171)$ \\
\hline IntConf & $-0.0617^{* * *}$ & -0.0131 & $-0.0544^{* * *}$ & -0.000538 \\
\hline & $(0.0137)$ & $(0.00905)$ & $(0.0201)$ & $(0.0151)$ \\
\hline RelTens & $-0.145^{* * *}$ & $-0.0280^{* *}$ & $-0.105^{* * *}$ & $-0.0304^{* *}$ \\
\hline & $(0.0206)$ & $(0.0140)$ & $(0.0301)$ & $(0.0231)$ \\
\hline LnSP & $3.935^{* * *}$ & $0.957^{* *}$ & $3.588^{* * *}$ & $0.293^{*}$ \\
\hline & $(0.626)$ & $(0.421)$ & $(0.919)$ & $(0.702)$ \\
\hline
\end{tabular}




\begin{tabular}{|l|l|l|l|l|}
\hline LnIPC & & $0.0484^{* *}$ & & -0.0434 \\
\hline & & $(0.0239)$ & & $(0.0394)$ \\
\hline LnGDP & & $0.337^{* * *}$ & & $0.705^{* * *}$ \\
\hline & & $(0.0479)$ & & $(0.0720)$ \\
\hline LnUP & & $0.970^{* * *}$ & & $1.047^{* * *}$ \\
\hline Constante & $1.910^{* * *}$ & $-3.016^{* * *}$ & $4.550^{* * *}$ & $-1.488^{*}$ \\
\hline Nombre de pays & 32 & $(0.0829)$ & & $(0.112)$ \\
\hline $\mathrm{R}^{2}$ & $(0.624)$ & $(0.615)$ & $(0.910)$ & $(0.922)$ \\
\hline Wald test (p-value) & 0,0000 & 0,0000 & 0,0000 & 0,0000 \\
\hline Observations & 704 & 682 & 704 & 682 \\
\hline & 0,1923 & 0,3918 & 0,1860 & 0,4007 \\
\hline
\end{tabular}

Les valeurs entre parenthèses sont des écarts-types.

*** $p<0.01, * \star p<0.05, * p<0.1$ les seuils de significativité.

Les modèles 1 et 2 du tableau 2 montrent que toutes les variables individuelles et la variable composite de la stabilité politique ont des effets respectivement négatifs et positifs significatifs sur le tourisme aussi bien en termes du nombre d'arrivées qu'en termes de recettes. Ces résultats montrent, toutes choses égales par ailleurs, que les tensions ethniques, les tensions religieuses, les conflits internes et externes réduisent significativement les arrivées des touristes et les recettes du tourisme. La stabilité politique affecte positivement et significativement le tourisme. Par exemple, une détérioration d'un point de l'indice de conflit interne conduit à une réduction de $61 \%$ du nombre de touristes et de $54 \%$ des recettes touristiques. Les résultats corroborent ceux de Yap et Saha (2013) qui montrent que l'instabilité politique réduit significativement les flux touristiques en raison de la contribution d'un état d'instabilité à l'image d'une destination.

Puisque les touristes consomment une combinaison de caractéristiques d'une destination (et non un seul bien), les variables de contrôle sont introduites pour tenir compte de l'influence des autres facteurs. Ces variables représentent les prix relatifs, le revenu par tête et le degré de similarité dans les modèles 3 et 4 . La significativité des coefficients positifs associés à toutes les variables de contrôle atteste du fait que les touristes choisissent des destinations qui présentent presque les mêmes caractéristiques que leur pays d'origine en termes de coût de vie, d'urbanisation et de revenu. 
Tableau 3. Effet de la qualité des institutions sur le tourisme (modèle à effets aléatoires)

\begin{tabular}{|c|c|c|c|c|}
\hline & (1) & (2) & (3) & $(4)$ \\
\hline Variables & LnITD & LnITD & LnITR & LnITR \\
\hline \multirow[t]{2}{*}{ BurQua } & $-0.0972^{* * *}$ & $0.0945^{* * *}$ & $-0.0839^{*}$ & $0.144^{* * *}$ \\
\hline & $(0.0343)$ & $(0.0244)$ & $(0.0496)$ & $(0.0406)$ \\
\hline \multirow[t]{2}{*}{ Corrup } & $-0.210^{* * *}$ & -0.0138 & $-0.274^{* * *}$ & -0.0442 \\
\hline & $(0.0287)$ & $(0.0206)$ & $(0.0417)$ & $(0.0346)$ \\
\hline \multirow[t]{2}{*}{ GovStab } & $-0.144^{* * *}$ & $0.0316^{*}$ & $-0.154^{* * *}$ & $0.0556^{*}$ \\
\hline & $(0.0261)$ & $(0.0186)$ & $(0.0378)$ & $(0.0312)$ \\
\hline \multirow[t]{2}{*}{ LawOrdI } & $-0.162^{* * *}$ & $0.0793^{* * *}$ & $-0.146^{* * *}$ & $0.134^{* * *}$ \\
\hline & $(0.0334)$ & $(0.0241)$ & $(0.0481)$ & $(0.0397)$ \\
\hline \multirow[t]{2}{*}{ DemoAcc } & $-0.0826^{* * *}$ & 0.0187 & -0.0641 & 0.0499 \\
\hline & $(0.0281)$ & $(0.0194)$ & $(0.0407)$ & $(0.0327)$ \\
\hline \multirow[t]{2}{*}{ LogQI } & $4.845^{* * *}$ & $1.008^{*}$ & $4.930^{* * *}$ & $1.900^{* *}$ \\
\hline & $(1.031)$ & $(0.723)$ & $(1.495)$ & $(1.218)$ \\
\hline \multirow[t]{2}{*}{ LnIPC } & & $0.0793^{* * *}$ & & 0.00359 \\
\hline & & $(0.0235)$ & & $(0.0388)$ \\
\hline \multirow[t]{2}{*}{ LnGDP } & & $0.325^{* * *}$ & & $0.687^{* * *}$ \\
\hline & & $(0.0472)$ & & $(0.0694)$ \\
\hline \multirow[t]{2}{*}{ LnUP } & & $1.023^{* * *}$ & & $0.983^{* * *}$ \\
\hline & & $(0.0822)$ & & $(0.103)$ \\
\hline \multirow[t]{2}{*}{ Constante } & $2.103^{* *}$ & $-1.810^{* *}$ & $4.767^{* * *}$ & 0.851 \\
\hline & $(0.837)$ & $(0.707)$ & $(1.210)$ & $(1.092)$ \\
\hline $\mathrm{R}^{2}$ & 0.1650 & 0.4690 & 0.1644 & 0.5010 \\
\hline Wald test (p-value) & 0,0000 & 0,0000 & 0,0000 & 0,0000 \\
\hline Observations & 704 & 682 & 704 & 682 \\
\hline
\end{tabular}




\begin{tabular}{|l|l|l|l|l|}
\hline Nombre de pays & 32 & 31 & 32 & 31 \\
\hline
\end{tabular}

Notes : Les valeurs entre parenthèses sont des écarts-types ${ }^{\star * \star} p<0.01,{ }^{* \star} p<0.05,{ }^{\star} p<0.1$ institutions et la demande internationale de tourisme, quelle que soit la spécification retenue. La qualité des institutions est constituée d'un ensemble de variables qui, lorsque prises individuellement, affectent négativement le tourisme. Par exemple, sur l'échantillon de pays africains considérés, une hausse de l'indice de la corruption fait chuter les recettes touristiques de $27 \%$ et réduit le nombre de touristes de $21 \%$. Un service public ne peut être efficace dans un système corrompu car cette inefficacité a pour conséquence d'augmenter les coûts de transaction. De même, une dégradation de la démocratie dans un pays réduit les arrivées touristiques de $8,26 \%$ et les recettes touristiques de $6,4 \%$. Bien que les touristes soient peu sensibles au régime politique en place, ils préfèrent les pays à régime démocratique car ils estiment que leurs droits y seront plus respectés. Ces résultats sont similaires à ceux de Yap et Saha (2013) et de Das et Dirienzo (2010). Il est intéressant de remarquer que les pays ayant une bonne qualité institutionnelle attirent plus de touristes (à l'exemple du Kenya et du Maroc, qui font partie des destinations les plus visitées) que les pays où la qualité institutionnelle est déplorable (comme le Soudan ou la République centrafricaine, qui attirent très peu de touristes, voire pas de touristes du tout). Ces résultats sont conformes à ceux de Kim et al. (2017) et de UNCTAD (2017).

Les coefficients associés aux variables de contrôle présentent tous les signes attendus. Le coefficient du PIB par tête est positif et significatif, ce qui montre l'importance du revenu dans la décision de voyager. Ainsi, une augmentation de $1 \%$ du revenu conduit à une hausse du nombre de touristes de $32,5 \%$ et de $68,7 \%$ des recettes du tourisme. Il en va de même du taux d'inflation et de l'urbanisation de la population qui affectent positivement et significativement les prix relatifs et le degré de similarité. Ces résultats restent robustes même si l'on change de variable dépendante.

Enfin, une variable d'interaction est introduite afin d'évaluer l'effet conjoint de la stabilité politique et de la qualité des institutions sur le tourisme. À l'issue des estimations, il apparait clairement que la stabilité politique et la qualité des institutions sont liées et ont des effets positifs sur le tourisme.

Tableau 4. Effets de la stabilité politique et de la qualité des institutions sur le tourisme (modèle à effets aléatoires)

\begin{tabular}{|l|l|l|}
\hline & $(\mathbf{1})$ & $(\mathbf{2})$ \\
\hline Variables & LnITD & LnITR \\
\hline & & \\
\hline LnIPC & $0.0735^{* * *}$ & -0.00550 \\
\hline & $(0.0236)$ & $(0.0395)$ \\
\hline LnGDP & $0.314^{* * *}$ & $0.680^{* * *}$ \\
\hline
\end{tabular}

Mondes du Tourisme, 19 | 2021 


\begin{tabular}{|c|c|c|}
\hline & $(0.0476)$ & $(0.0684)$ \\
\hline \multirow[t]{2}{*}{ LnUP } & $1.031^{* * *}$ & $0.990^{* * *}$ \\
\hline & $(0.0834)$ & (0.0975) \\
\hline \multirow[t]{2}{*}{ LnSP } & $3.443^{* * *}$ & $3.952^{*}$ \\
\hline & $(1.227)$ & $(2.105)$ \\
\hline \multirow[t]{2}{*}{ LnQI } & $4.091^{* * *}$ & $5.487^{* *}$ \\
\hline & $(1.440)$ & $(2.469)$ \\
\hline \multirow[t]{2}{*}{$\operatorname{LnSP} \times \ln Q I$} & $2.969^{* * *}$ & $3.866^{* *}$ \\
\hline & $(1.005)$ & $(1.722)$ \\
\hline \multirow[t]{2}{*}{ Constante } & 2.305 & $5.177^{*}$ \\
\hline & $(1.776)$ & $(3.015)$ \\
\hline Wald test ( $p$-value) & 0,0000 & 0,0000 \\
\hline $\mathrm{R}^{2}$ & 0.4067 & 0.4599 \\
\hline Observations & 682 & 682 \\
\hline Nombre de pays & 31 & 31 \\
\hline
\end{tabular}

Notes : Les valeurs entre parenthèses sont des écarts-types.

$\star \star \star * ~ p<0.01,{ }^{* *} p<0.05,{ }^{\star} p<0.1$

Les résultats reportés dans le tableau 4 affichent un coefficient de la variable d'interaction positif et significatif, ce qui témoigne d'un effet positif conjoint de la stabilité politique et de la qualité des institutions sur le tourisme. Généralement, les pays instables ont des institutions faibles dans la mesure où les politiques sont difficilement menées à leur terme. Les réglementations et les décisions politiques ne sont pas respectées du fait du manque de suivi et de rigueur au sein des institutions. Les gouvernements y sont instables et la constitution utilisée à des fins personnelles. A contrario, un pays stable a tendance à renforcer la qualité de ses institutions, d'où cette interaction positive entre la stabilité politique et la qualité des institutions. Ainsi, une amélioration d'un point de l'indice de l'interaction entre la stabilité politique et la qualité des institutions entraîne une hausse du nombre de touristes de $296,9 \%$ et une hausse des recettes touristiques de $386,6 \%$. Un pays stable peut donc, à long terme, conduire à une amélioration progressive de la qualité des institutions et gagner rapidement des parts de marché en termes d'attractivité touristique. 


\subsection{Contrôle de l'endogénéité}

51 La littérature sur le sens de causalité de la stabilité politique et du tourisme semble mitigée. Pour les uns (Causevic et Lynch, 2011 ; UNCTAD, 2017 ; Pratt et Liu, 2017), le tourisme est un excellent moyen de réunir les peuples, donc un moyen de conduire à la stabilité politique et à la paix. Pour les autres (Chingarande, 2014 ; Hall et O'Sullivan, 1996; Nyaruwata, 2013; Neumayer, 2004), c'est la stabilité politique qui permet d'attirer les touristes. Dès lors, se pose un problème de biais d'endogénéité. Face à ce problème, les estimateurs des moindres carrés généralisés seront biaisés lorsque la corrélation entre le terme d'erreur et les deux variables d'intérêt est importante. En vue de traiter le biais potentiel d'endogénéité, nous utilisons l'estimateur GMM en différence d'Arellano et Bond (1991). Cet estimateur présente l'avantage d'une différence première éliminant l'effet spécifique pays et, par conséquent, le biais de variables omises invariantes dans le temps. Les valeurs retardées ${ }^{8}$ de toutes les variables sont utilisées comme instruments. Les tests de Sargan/Hansen ${ }^{9}$ permettent de valider les instruments utilisés. Les résultats des estimations de GMM sont présentés dans le tableau 5. Pour confirmer nos résultats, nous avons effectué quelques tests de robustesse à l'aide des indicateurs WGI, dont les résultats sont présentés à l'annexe 4.

Tableau 5. Effets de la stabilité politique et de la qualité des institutions sur le tourisme (GMM)

\begin{tabular}{|l|l|l|}
\hline & $(\mathbf{1})$ & $(\mathbf{2})$ \\
\hline Variables & LnITD & LnITR \\
\hline & & \\
\hline LnITD $_{\mathrm{t}-1}$ & $0.518^{* * *}$ & \\
\hline & $(0.0560)$ & \\
\hline LnIPC & $-0.0438^{* * *}$ & $0.0694^{* * *}$ \\
\hline & $(0.0121)$ & $(0.0169)$ \\
\hline LnGDP & $0.117^{* * *}$ & $0.388^{* * *}$ \\
\hline & $(0.0167)$ & $(0.0553)$ \\
\hline LnUP & $0.685^{* * *}$ & $0.844^{* * *}$ \\
\hline & $(0.0935)$ & $(0.122)$ \\
\hline LnSP & $-5.419^{* * *}$ & -0.840 \\
\hline & $(1.435)$ & $(0.655)$ \\
\hline LnQI & $-6.793^{* * *}$ & $-1.663^{*}$ \\
\hline & $(1.827)$ & $(0.817)$ \\
\hline LnSP*LnQI & $4.742^{* * *}$ & $1.145^{*}$ \\
\hline
\end{tabular}




\begin{tabular}{|l|l|l|}
\hline & $(1.264)$ & $(0.588)$ \\
\hline LnITR $_{\mathrm{t}-1}$ & & $0.231^{* * *}$ \\
\hline & & $(0.0657)$ \\
\hline & & \\
\hline Sargan/Hansen & 0.321 & 0,244 \\
\hline Instruments & 96 & 26 \\
\hline Observations & 620 & 620 \\
\hline Nombre de pays & 31 & 31 \\
\hline
\end{tabular}

Les valeurs entre parenthèses sont des écart-types.

$\star \star * *<0.01,{ }^{* \star} p<0.05,{ }^{\star} p<0.1$ sont les seuils de significativité.

Globalement, les résultats obtenus à partir des GMM en différence restent cohérents avec ceux obtenus à l'aide du modèle à effets aléatoires, confirmant de ce fait l'effet positif de la stabilité politique et de la qualité des institutions sur la demande internationale de tourisme. Les résultats de l'analyse suggèrent une association positive de la stabilité politique, de la qualité des institutions et de leur interaction sur le tourisme international. La stabilité politique, en réduisant l'incertitude et l'insécurité, permet de donner une bonne image de la destination. Tout touriste, en optant pour une destination, se projette dans un environnement stable et agréable, où sa sécurité ne sera pas menacée. Ainsi, tout pays qui désire attirer plus de touristes doit s'assurer de réduire les risques susceptibles de nuire au bien-être des touristes et à l'image de la destination. De plus, il doit s'organiser de manière à améliorer la qualité de ses institutions en réduisant les délais d'obtention des papiers nécessaires, les coûts de transaction et en protégeant les intérêts des touristes et des investisseurs du secteur touristique. En comparant les résultats des tableaux 4 et 5 , on peut affirmer de manière confiante qu'une amélioration marginale $(+1 \%)$ de l'interaction entre la stabilité politique et la qualité des institutions assure une multiplication par 6 à 8 du nombre de touristes pour, au minimum, un doublement des recettes touristiques internationales.

\section{Recommandations}

$53 \mathrm{Au}$ regard de nos résultats, quelques recommandations en termes de politiques économiques se dégagent.

Premièrement, les gouvernements en Afrique doivent mettre un accent particulier sur la stabilité politique afin qu'une bonne qualité institutionnelle s'ensuive. L'amélioration de la stabilité politique et de la qualité des institutions relèvent de la volonté politique de créer des institutions dignes de confiance tournées vers les intérêts collectifs nationaux.

Deuxièmement, les pays africains qui souhaitent développer leur secteur touristique sont censés, au préalable, gagner la confiance des touristes en leurs destinations. Pour ce faire, des actions concrètes quant à la sécurité des touristes et des sites touristiques 
sont requises. Ils doivent par ailleurs faciliter les procédures d'octroi de visas pour des cibles touristiques bien identifiées, réduire leurs coûts de transaction en mettant en œuvre des réformes adéquates pour éradiquer la mal-gouvernance.

Troisièmement, les pays doivent investir dans l'aménagement des sites touristiques et construire des infrastructures. En effet, l'industrie touristique dépend de plusieurs activités connexes qui nécessitent des investissements dans un environnement stable et sécurisé, avec un effet multiplicateur sur le reste de l'économie non négligeable.

Quatrièmement, la lutte contre la corruption doit être une bataille quotidienne, puisque la corruption a pour effet d'augmenter les coûts de transactions ; or, comme les touristes tiennent compte des prix relatifs, elle constitue un frein au développement du tourisme.

\section{Conclusion}

Cet article a cherché à évaluer l'effet de l'interaction entre la stabilité politique et la qualité des institutions sur la demande effective de tourisme international. Pour atteindre cet objectif, nous avons d'abord analysé l'effet de la stabilité politique sur le tourisme, puis l'effet de la qualité des institutions sur le tourisme, avant de considérer l'effet de l'interaction entre la stabilité politique et la qualité des institutions sur le tourisme international. L'analyse a porté sur un échantillon de 32 pays africains sur la période 1996-2017. Les résultats des estimations issus du modèle à effets aléatoires et des GMM ne rejettent pas les hypothèses selon lesquelles la stabilité politique et la qualité des institutions sont chacune positivement corrélées à la demande de tourisme international. L'interaction entre la stabilité politique et la qualité des institutions a également un impact positif et significatif sur la demande internationale de tourisme. En d'autres termes, la stabilité politique, la qualité des institutions et l'interaction entre elles sont à même de favoriser une forte augmentation de la demande touristique dans les pays africains. Ces résultats demeurent robustes à toutes les spécifications.

Il serait intéressant à l'avenir de déterminer à partir de quel seuil la stabilité politique et la qualité des institutions influencent la demande internationale de tourisme.

\section{BIBLIOGRAPHIE}

Emmanuel Akwasi ADU-AMPONG, «The Tourism-Development Nexus from a Governance Perspective: A Research Agenda ", dans Richard Sharpley et David Harrison (dir.), A research Agenda for Tourism and Development, Edward Elgar Publishing, 2019, p. 53-70 [https://doi.org/ 10.4337/9781788112413.00009].

Emmanuel Akwasi ADU-AMPONG, « Divided We Stand: Institutional Collaboration in Tourism Planning and Development in the Central Region of Ghana ", Current Issues in Tourism, vol. 20, $\mathrm{n}^{\circ}$ 3, p. 295-314, 2017 [https://doi.org/10.1080/13683500.2014.915795]. 
Emmanuel Akwasi ADU-AMPONG, Marina NOVELLI et Manuel Alector RIBEIRO, « Tourism in AfricaContinental and Regional Contexts ", dans Emmanuel Akwasi Adu-Ampong, Marina Novelli et Manuel Alector Ribeiro (dir.), Handbook of Tourism in Africa, Routledge, 2020.

Maria Dolores ALVAREZ et Sara CAMPO, « The Influence of Political Conflicts on Country Image and Intention to visit: A Study of Israel's Image », Tourism Management, vol. 40, p. 70-78, 2014 [https:// doi.org/10.1016/j.tourman.2013.05.009].

Manuel ARELLANo et Stephen BOND, « Some Tests of Specification for Panel Data: Monte Carlo Evidence and an Application to Employment Equations ", Review of Economic Studies, vol. 58, $\mathrm{n}^{\circ} 2$, p. 277-297, 1991 [https://doi.org/10.2307/2297968].

Simplice A. ASONGU et Jacinta C. NWACHUKWU, « The Impact of Terrorism on Governance in African Countries », World Development, n 99, p. 253-270, 2017 [https://doi.org/10.1016/j.worlddev. 2017.05.023].

Simplice A. ASONGU, Joseph NNANNA, Nicholas BIEKPE et Paul N. ACHA-ANYI, « Contemporary Drivers of Global Tourism: Evidence from Terrorism and Peace Factors ", Journal of Travel \& Tourism Marketing, vol. 36, n 3, p. 345-357, 2019 [https://doi.org/10.1080/10548408.2018.1541778].

Badi H. BALTAGI, Econometric Analysis of Panel Data, Wiley, 2008 (4 ${ }^{\text {ème }}$ ed.).

BBC NEWS, « How Terrorist Attacks Affect Tourism », 29 juin, 2015 [https://www.bbc.com/news/ magazine-33310217.amp].

BBC NEWS, « Egypt in Numbers », 24 mai, 2014 [https://www.bbc.com/news/world-middleeast-27251107].

De Shahid Javed BURKI et Guillermo PERRY, Beyond the Washington Consensus: Institutions Matter, World Bank Latin American and Caribbean Studies, World Bank Publication, 1998.

Senija CAUSEVIC et Paul LYNCH, « Political (In)Stability and its Influence on Tourism Development », Tourism Management, vol. 34, n 1, p. 145-157, 2013 [https://doi.org/10.1016/j.tourman.

2012.04.006].

Senija CAUSEVIC et Paul LYNCH, «Phoenix Tourism: Post Conflict Tourism Role », Annals of Tourism Research, vol. 38, n 3, p. 780-800, 2011 [https://doi.org/10.1016/j.annals.2010.12.004].

Anna CHINGARANDE, « Does Political Instability Affect Tourism: a Case of Egypt », International Journal of Social Relevance Concern, vol. 2, n 6, 2014.

Carl J. DAHLMAN, «The Problem of Externality », The Journal of Law and Economics, vol. 22, $\mathrm{n}^{\circ} 1$, p. 141-162, 1979 [https://doi.org/10.1086/466936].

Jayoti DAS et Cassandra DIRIENZO, « Tourism Competitiveness and Corruption: A Cross-Country Analysis », Tourism Economics, vol. 16, n³, p. 477-492, 2010 [https://doi.org/ 10.5367\%2F000000010792278392].

Jayoti DAS et Cassandra DIRIENZO, « Global Tourism Competitiveness and Freedom of the Press: A Nonlinear Relationship ", Journal of Travel Research, vol. 47, n 4, p. 470-479, 2009 [https://doi.org/ 10.1177\%2F0047287508326535].

Yair EILAT et Liran EINVA, « Determinants of International Tourism: A Three-Dimensional Panel Data Analysis », Applied Economics, vol. 36, n 12, p. 1315-1327, 2004 [https://doi.org/ 10.1080/000368404000180897]. 
Ibrahim ELSHAER A. et Samar K. SAAD, « Political Instability and Tourism in Egypt: Exploring Survivors Attitude After Downsizing », Journal of Policy Research in Tourism, Leisure and Events, vol. 9, n 1 p. 3-22, 2017 [https://doi.org/10.1080/19407963.2016.1233109].

Walter ENDERS et Todd SANDLER, « Causality Between Transnational Terrorism and Tourism: The Case of Spain », Terrorism, vol. 14, n 1, p. 49-58, 1991 [https://doi.org/ $10.1080 / 10576109108435856]$.

Walter ENDERS, Todd SANDLER et Gerald PARISE, « An Econometric Analysis of the Impact of Terrorism on Tourism », Kyklos, vol. 45, nº 4, p. 531-554, 1992.

Mete FERIDUN, « Impact of Terrorism on Tourism in Turkey: Empirical Evidence from Turkey », Applied Economics, vol. 43, n 24, p. 3349-3354, 2011 [https://doi.org/10.1080/00036841003636268]. Giray GOZGOR et Serdar ONGAN, « Economic Policy Uncertainty and Tourism Demand: Empirical Evidence from the USA », International Journal of Tourism Research, vol. 19, p. 99-106, 2017 [https:// doi.org/10.1002/jtr.2089].

Colin Michael HaLl et Vincent o'sulLIVAN, « Tourism, Political Stability and Violence ", dans Abraham Pizam et Yoel Mansfeld (dir.), Tourism, Crime and International Security Issues, Wiley, 1996. Hadryn IMGRAM, Saloomeh TABARI et Wanthmee WATTHANAKHOMPRATHIP, « The Impact of Political Instabiliy on Tourism: Case of Thailand ", Worldwide Hospitality and Tourism Themes, vol. 5, $\mathrm{n}^{\circ} 1$, p. 92-103, 2013 [https://doi.org/10.1108/17554211311292475].

IEP, Global Peace Index 2019: Measuring Peace in a Complex World, Institute for Economics \& Peace, 2019 [https://www.visionofhumanity.org/wp-content/uploads/2020/10/GPI-2019web.pdf].

Stanislav IVANOV, Margaret GAVRILINA, Craig WEBSTER et Vladyslay RALKo, « Impacts of Political Instability on the Tourism Industry in Ukraine ", Journal of Policy Research in Tourism, Leisure and Events, vol. 9, n 1, p. 100-127, 2017 [https://doi.org/10.1080/19407963.2016.1209677].

Kun KIM, Oun Joung PARK, Seunghyun YUN et Heajung YUN, « What Makes Tourists Feel Negatively about Tourism Destinations? Application of Hybrid Text Mining Methodology to Smart Destination Management ", Technological Forecasting and Social Change, vol. 123, p. 362-369, 2017 [https://doi.org/10.1016/j.techfore.2017.01.001].

Seongseop KIM, Ja Young CHOE et Suna LEE, « How are Food Value Video Clips Effective in Promoting Food Tourism? Generation Y versus Non-Generation Y ", Journal of Travel \& Tourism Marketing, vol. 35 n 3, p. 377-393, 2018 [https://doi.org/10.1080/10548408.2017.1320262].

Theodore LEVANTIS et Azmat GANI, « Tourism Demand and the Nuisance of Crime ", International Journal of Social Economics, vol. 27, n 7, 8, 9, 10, p. 959-967, 2000 [https://doi.org/ $10.1108 / 03068290010336964]$.

Anyu LIU et Stephen PRATT, « Tourism's Vulnerability and Resilience to Terrorism », Tourism Management, vol. 60, p. 404-417, 2017 [https://doi.org/10.1016/j.tourman.2017.01.001].

Rafael LLORCA-VIVERO, « Terrorism and International Tourism: New Evidence ", Defence and Peace Economics, vol. 19, n² 2, p. 169-188, 2008 [https://doi.org/10.1080/10242690701453917].

Chrysostomos MANTZAVINos, Individuals, Institutions, and Markets, Cambridge University Press, 2001.

Harry G. MATTHEWS, « International Tourism and Political Science Research », Annals of Tourism Research, vol. 2, n 4, p. 195-203, 1974 [https://doi.org/10.1016/0160-7383(75)90032-8].

Jan MOSEDALE, « Political Economy of Tourism », dans Alan A. Lew, Michael C. Hall et Allan M. Williams (dir.), The Wiley Blackwell Companion to Tourism (Chap. 4), Blackwell, 2014, p. 55-65. 
Eric NEUMAYER, « The impact of political violence on tourism ", Journal of Conflict Resolution, vol. 48, $\mathrm{n}^{\circ}$ 2, p. 259-281, 2004 [https://doi.org/10.1177\%2F0022002703262358].

Eric NEUMAYER et Thomas PLÜMPER, « Spatial Spill-Overs from Terrorism on Tourism: Western Victims in Islamic Destination Countries », Public Choice, vol. 169, n³-4, p. 195-206, 2016.

Eric NEUMAYER et Thomas PLÜMPER, «International Terrorism and the Clash of Civilizations », British Journal of Political Science, vol. 39, n 4, p. 711-734, 2009 [https://www.jstor.org/stable/ 27742769].

Douglass C. NORTH et Robert Paul THOMAS, The Rise of the Western World: A New Economic History, Cambridge University Press, 1973.

Robin NUNKOO, Haywantee RAMKISSOON et Dogan GURSOY, « Public Trust in Tourism Institutions ", Annals of Tourism Research, vol. 39, n³, p. 1538-1564, 2012 [https://doi.org/10.1016/j.annals. 2012.04.004].

Shepherd NYARUWATA, Alick MHIZHA et Golden MANDEBVU, « Impact of Political Instability on Tourism Development: A comparative Analysis of Mauritius and Zimbabwe since Independence », Journal of Tourism and Peace Research, vol. 3, n² 2, p. 43-56, 2013.

OMT, Faits saillants du tourisme, publication de l'Organisation mondiale du tourisme, 2017.

Arian PALMER et David BEJOU, « Tourism Destination Marketing Alliances », Annals of Tourism Research, vol. 22, n 3, p. 616-629, 1995 [https://doi.org/10.1016/0160-7383(95)00010-4].

Thomas PLÜMPER et Eric NEUMAYER, «The Friend of My Enemy is My Enemy: International Alliances and International Terrorism ", European Journal of Political Research, vol. 49, n 1 , p. 75-96, 2010 [https://doi.org/10.1111/j.1475-6765.2009.01885.x].

Stephen PRATT et Anyu LIU, « Tourism's Vulnerability and Resilience to Terrorism », Tourism Management, vol. 60, p. 404-417, 2017 [https://doi.org/10.1016/j.tourman.2017.01.001].

Stephen PRATT et Anyu LIU, « Does Tourism Really Lead to Peace? A Global View », International Journal of Tourism Research, vol. 18, n 1, p. 82-90, 2016 [https://doi.org/10.1002/jtr.2035].

Natalia RESTREPO et Salvador Anton CLAVÉ, « Institutional Thickness and Regional Tourism Development: Lessons from Antioquia, Colombia », Sustainability, vol. 11, n 9, 2019, [https:// doi.org/10.3390/su11092568].

Linda K. RICHTER et William L. WAUGH, « Terrorism and Tourism as Logical Companions », Tourism Management, vol. 7, p. 230-238, 1986 [https://doi.org/10.1016/0261-5177(86)90033-6].

Shrabani SAHA et Ghaily YAP, « Corruption and Tourism: An Empirical Investigation in a NonLinear Framework », International Journal of Tourism Research, vol. 17, p. 272-281, 2015 [https:// doi.org/10.1002/jtr.1985].

Shrabani SAHA et Ghaily YAP, « The Moderation Effects of Political Instability and Terrorism on Tourism Development », Journal of Travel Research, vol. 53, n 4, p. 509-521, 2014 [https://doi.org/ 10.1177\%2F0047287513496472].

Shrabani SAHA, Jen Je su et Niel CAMPBELL, « Does Political and Economic Freedom Matter for Inbound Tourism? A Cross-National Panel Data Estimation », Journal of Travel Research, vol. 56, n 2, p. 221-234, 2017 [https://doi.org/10.1177\%2F0047287515627028].

Richard W. scoTT, Institutions and Organizations. Foundations for Organizational Science, Sage Publications, 1995.

Thea M. SINCLAIR et Mike J. STABLER, The Economics of Tourism, Routledge, 1997. 
Sevil F. sÖNMEZ, « Tourism, Terrorism, and Political Instability », Annals of Tourism Research, vol. 25, n² 2, p. 416-456, 1998 [https://doi.org/10.1016/S0160-7383(97)00093-5].

Sevil F. sönMEZ, Yiorgos APOSTOLOPOUlos et Peter TARLow, « Tourism in Crisis: Managing the Effects of Terrorism », Journal of Travel Research, vol. 38, p. 13-18, 1999 [https://doi.org/ 10.1177\%2F004728759903800104].

Yu-Wen su et Hui Lin LIN, « Analysis of International Tourist Arrivals Worldwide: The Role of World Heritage Sites », Tourism Management, vol. 40, p. 46-58, 2014 [https://doi.org/10.1016/ j.tourman.2013.04.005].

Chor Foon TANG, « The Impacts of Governance and Institutions on Inbound Tourism Demand: Evidence from a Dynamic Panel Data Study ", Asia Pacific Journal of Tourism Research, vol. 23, $\mathrm{n}^{\circ} 10$, p. 1000-1007, 2018 [https://doi.org/10.1080/10941665.2018.1513052].

UNCTAD, Economic Development in Africa Report 2017: Tourism for Transformative and Inclusive Growth, United Nations Conference on Trade and Development, United Nations publication, 2017.

UNWTO, UNWTO Annual Report 2019, United Nations World Tourism Organization, 2019.

UNWTO, UNWTO Annual Report 2017, United Nations World Tourism Organization, 2018.

UNWTO, UNWTO Annual Report 2016, United Nations World Tourism Organization, 2017.

UNWTO, UNWTO Tourism Highlights, United Nations World Tourism Organization, 2016a [http:// www.e-unwto.org/doi/pdf/10.18111/9789284418145].

UNWTO, UNWTO Annual Report 2015, United Nations World Tourism Organization, 2016b.

UNWTO, UNWTO Annual Report 2014, United Nations World Tourism Organization, 2015.

UNWTO, UNWTO Annual Report 2013, United Nations World Tourism Organization, 2014.

Mathilda VAN NIEKERK, « The Role of the Public Sector in Tourism Destination Management from a Network Relationship Approach », Tourism Analysis, vol. 19, n 6, p. 701-718, 2014 [https:// doi.org/10.3727/108354214X14146846679402].

Oliver E. WILLIAMSON, « Transaction-Cost Economics: The Governance of Contractual Relations ", Journal of Law and Economics, vol. 2, p. 233-261, 1979.

Ghaily YAP et Shrabani SAHA, « Do Political Instability, Terrorism, and Corruption Have Deterring Effects on Tourism Development Even in the Presence of UNESCO Heritage? A Cross-Country Panel Estimate », Tourism Analysis, vol. 18, p. 587-599, 2013 [https://doi.org/

10.3727/108354213X13782245307911].

\section{ANNEXES}

\section{Annexe 1. Liste des pays}

Cameroun, Congo, Gabon, République démocratique du Congo, Kenya, Madagascar, Malawi, Malaisie, Mozambique, Soudan, Ouganda, Burkina-Faso, Côte d'Ivoire, Gambie, Ghana, Guinée-Bissau, Mali, Niger, Nigeria, Sierra Leone, Sénégal, Togo, Afrique du Sud, Angola, Botswana, Zambie, Zimbabwe, Algérie, Égypte, Libye, Maroc, Tunisie. 


\section{Annexe 2. Matrice de corrélation entre les variables institutionnelles}

\begin{tabular}{|l|l|l|l|l|l|l|l|l|l|l|l|l|}
\hline Variables & $(1)$ & $(2)$ & $(3)$ & $(4)$ & $(5)$ & $(6)$ & $(7)$ & $(8)$ & $(9)$ & $(10)$ & $(11)$ & $(12)$ \\
\hline (1) Burqua & 1.000 & & & & & & & & & & & \\
\hline$(2)$ Corrup & 0.180 & 1.000 & & & & & & & & & & \\
\hline (3) GovSta & -0.080 & 0.145 & 1.000 & & & & & & & & & \\
\hline (4) LawOrd & 0.300 & 0.323 & 0.208 & 1.000 & & & & & & & & \\
\hline (5) DemoAcc & 0.231 & 0.187 & -0.131 & 0.106 & 1.000 & & & & & & & \\
\hline (6) EthTens & 0.381 & 0.267 & 0.112 & 0.625 & 0.107 & 1.000 & & & & & & \\
\hline (7) ExtConf & 0.259 & 0.241 & 0.136 & 0.129 & 0.327 & 0.306 & 1.000 & & & & & \\
\hline (8) IntCon & 0.183 & 0.289 & 0.224 & 0.402 & 0.228 & 0.468 & 0.533 & 1.000 & & & & \\
\hline $\begin{array}{l}\text { (10) RelTen } \\
\text { (11) LnSP }\end{array}$ & 0.081 & 0.270 & 0.113 & 0.311 & 0.181 & 0.281 & 0.194 & 0.459 & 0.312 & 1.000 & & \\
\hline somme & 0.359 & 0.169 & 0.497 & 0.358 & 0.673 & 0.693 & 0.848 & 0.745 & 0.597 & 1.000 & \\
\hline $\begin{array}{l}\text { (12) } \\
\text { somme }\end{array}$ & 0.430 & 0.566 & 0.606 & 0.650 & 0.443 & 0.487 & 0.384 & 0.479 & 0.502 & 0.306 & 0.590 & 1.000 \\
\hline
\end{tabular}

\section{Annexe 3. Description des variables}

\begin{tabular}{|l|l|l|l|}
\hline Variables & Définitions & Sources & Abréviations \\
\hline $\begin{array}{l}\text { Demande } \\
\text { internationale de } \\
\text { tourisme }\end{array}$ & $\begin{array}{l}\text { Mesurée par: } \\
\text { le nombre de touristes internationaux entrants } \\
\text { les recettes issues du tourisme international en } \\
\text { dollars US }\end{array}$ & WDI & ITD \\
\hline Revenu & Mesuré par le produit intérieur brut par tête & WDI & GDP \\
\hline $\begin{array}{l}\text { Inflation } \\
\text { Qualité } \\
\text { infrastructures et } \\
\text { degré de similarité } \\
\text { des pays }\end{array}$ & Mesurée par la population urbaine & WDI & IPC \\
\hline
\end{tabular}




\begin{tabular}{|c|c|c|c|}
\hline Corruption & $\begin{array}{l}\text { Indice de corruption au sein du système politique. Il } \\
\text { varie de } 0 \text { (forte corruption) à } 6 \text { (faible corruption). }\end{array}$ & ICRG & Corrup \\
\hline $\begin{array}{l}\text { Qualité de la } \\
\text { bureaucratie }\end{array}$ & $\begin{array}{l}\text { Indice de qualité de la bureaucratie, compris entre } 0 \\
\text { et } 4 \text {. Il évalue la qualité des services fournis par } \\
\text { l'administration publique (délais de livraison, } \\
\text { mécanismes de résolution des conflits) }\end{array}$ & ICRG & BurQua \\
\hline Ordre et loi & $\begin{array}{l}\text { Indice qui renseigne sur la présence d'institutions qui } \\
\text { préservent le droit de la propriété privée. Il fournit } \\
\text { une évaluation de la force et de l'impartialité du } \\
\text { système juridique, ainsi que du respect des droits } \\
\text { fondamentaux et de la loi. Il est compris entre } 0 \text { et } 6 \text {. }\end{array}$ & ICRG & Laword \\
\hline Démocratie & $\begin{array}{l}\text { Indice qui mesure non seulement la capacité du } \\
\text { gouvernement à conduire des élections libres et } \\
\text { transparentes, mais aussi sa capacité à répondre aux } \\
\text { besoins de sa population. Il est compris entre } 0 \text { et } 6 \text {. }\end{array}$ & ICRG & Demoacc \\
\hline $\begin{array}{l}\text { Stabilité } \\
\text { gouvernementale }\end{array}$ & $\begin{array}{l}\text { Cet indice, compris entre } 0 \text { et } 12 \text {, est calculé comme la } \\
\text { somme de trois sous-composantes, chacune mesurée } \\
\text { sur une échelle de } 0 \text { à } 4 \text { : unité gouvernementale, } \\
\text { force législative et soutien populaire. }\end{array}$ & ICRG & GovStab \\
\hline Stabilité politique & $\begin{array}{l}\text { Indice qui mesure la stabilité politique d'un pays sur } \\
\text { une base comparable avec d'autres pays en évaluant } \\
\text { les points de risque pour chacun des facteurs } \\
\text { suivants: stabilité gouvernementale, conditions } \\
\text { socioéconomiques, profil d'investissement, conflit } \\
\text { interne, conflit externe, corruption, politique } \\
\text { militaire, religion, tensions, loi et ordre, tensions } \\
\text { ethniques, responsabilité démocratique et qualité de } \\
\text { la bureaucratie. Cet indice varie de } 0 \text { (risque le plus } \\
\text { élevé) à } 100 \text { (risque le plus faible). }\end{array}$ & ICRG & $\begin{array}{l}\text { Ethtens, } \\
\text { ExtConf } \\
\text { Intconf } \\
\text { Reltens }\end{array}$ \\
\hline
\end{tabular}

\section{Annexe 4. Effet de la stabilité politique et de la qualité des institutions sur le tourisme : les indicateurs du WGI}

\begin{tabular}{|l|l|l|l|l|}
\hline & \multicolumn{2}{|l|}{ Effets aléatoires } & \multicolumn{2}{l|}{ GMM } \\
\hline & $(1)$ & $(2)$ & $(3)$ & $(4)$ \\
\hline Variables & LnITD & LnITR & LnITD & LnITR \\
\hline & & & & \\
\hline LnIPC & $0.0473^{*}$ & -0.0577 & 0.0303 & $0.249^{* *}$ \\
\hline
\end{tabular}




\begin{tabular}{|c|c|c|c|c|}
\hline & $(0.0277)$ & $(0.0443)$ & $(0.0573)$ & $(0.107)$ \\
\hline \multirow[t]{2}{*}{ LnGDP } & $0.259^{* * *}$ & $0.637^{* * *}$ & 0.0402 & $0.340^{* * *}$ \\
\hline & $(0.0514)$ & $(0.0761)$ & $(0.0298)$ & $(0.0711)$ \\
\hline \multirow[t]{2}{*}{ LnUP } & $1.112^{* * *}$ & $1.072^{* * *}$ & $0.571^{* * *}$ & 0.136 \\
\hline & $(0.0898)$ & $(0.119)$ & $(0.0757)$ & $(0.112)$ \\
\hline \multirow[t]{2}{*}{$\mathrm{SP} /$ noviolence } & $0.0559 * * *$ & 0.00656 & $0.0150^{* *}$ & $0.102^{* * *}$ \\
\hline & $(0.0213)$ & $(0.0344)$ & $(0.00686)$ & $(0.0171)$ \\
\hline \multirow[t]{2}{*}{ QI } & $0.0165^{*}$ & $0.0342^{* *}$ & $0.0212^{* * *}$ & $-0.0400^{* * *}$ \\
\hline & $(0.00929)$ & $(0.0145)$ & $(0.00479)$ & $(0.0106)$ \\
\hline \multirow[t]{2}{*}{$\operatorname{LnITD}_{\mathrm{t}-1}$} & & & $0.533^{* * *}$ & \\
\hline & & & $(0.0773)$ & \\
\hline \multirow[t]{2}{*}{ LnITRt- $_{1}$} & & & & $0.316^{* * *}$ \\
\hline & & & & $(0.0336)$ \\
\hline \multirow[t]{2}{*}{ Constante } & $-2.642 * * *$ & -0.571 & & \\
\hline & $(0.510)$ & $(0.682)$ & & \\
\hline Wald test (p-value) & 0.0000 & 0,0000 & & \\
\hline $\mathrm{R}^{2}$ & 0.4403 & 0.5045 & & \\
\hline Sargan/Hansen test ( $p$-value) & & & 0.470 & 0.203 \\
\hline Instruments & & & 25 & 25 \\
\hline Observations & 589 & 589 & 465 & 465 \\
\hline Nombre de pays & 31 & 31 & 31 & 31 \\
\hline
\end{tabular}

Notes : les valeurs entre parenthèses sont des écarts-types.

$* * * \mathrm{p}<0.01,{ }^{* *} \mathrm{p}<0.05,{ }^{*} \mathrm{p}<0.1$

\section{NOTES}

1. Cameroun, Congo, Gabon, République démocratique du Congo, Kenya, Madagascar, Malawi, Malaisie, Mozambique, Soudan, Ouganda, Burkina-Faso, Côte d'Ivoire, Gambie, Ghana, Guinée- 
Bissau, Mali, Niger, Nigeria, Sierra Léone, Sénégal, Togo, Afrique du Sud, Angola, Botswana, Zambie, Zimbabwe, Algérie, Égypte, Libye, Maroc, Tunisie.

2. Les institutions formelles désignent une réglementation et des règles juridiques, tandis que les institutions informelles sont des conventions, des programmes et des pratiques comportementaux légitimement et largement acceptés, qui guident les relations sociales et économiques ordinaires (Kim et al., 2018).

3. Ce test permet de tester la présence d'effets fixes.

4. Il permet de tester l'hypothèse d'absence d'effets individuels.

5. Le test de Hausman suit une loi de khi-Deux à K-1 degré de liberté et permet de faire un choix entre le modèle à effets fixes et le modèle à effets aléatoires.

6. InITR est la variable dépendante alternative.

7. La demande internationale de tourisme émane des personnes qui se rendent dans un pays autre que leur pays de résidence habituel pour une nuitée au moins et pour une période inférieure à 12 mois au plus, et qui ne travaillent pas pour une entreprise domiciliée dans le pays de destination.

8. Retardées d'une période pour les variables explicatives et de plus de deux périodes pour la variable dépendante.

9. $C f$. tableau 5 .

\section{RÉSUMÉS}

L'objectif de ce travail est d'évaluer l'effet de l'interaction entre la stabilité politique et la qualité des institutions sur la demande effective de tourisme international dans 32 pays africains sur la période 1996-2017. Pour ce faire, un modèle de données de panel à effets aléatoires est utilisé. L'estimateur de la méthode des moments généralisés (generalized method of moments, GMM) en différence est utilisé pour traiter tout biais potentiel d'endogénéité. Les résultats des estimations révèlent que la stabilité politique, la qualité des institutions et leur interaction ont respectivement des effets positifs et significatifs sur le tourisme. Ces résultats sont robustes à différentes spécifications compte tenu d'une diversité de mesures de la stabilité politique et de la qualité des institutions. De ce fait, les pays africains désireux de développer leur secteur touristique doivent chercher à améliorer leur stabilité politique et la qualité de leurs institutions. La stabilité politique et la qualité des institutions sont des conditions nécessaires mais pas suffisantes pour l'afflux des touristes.

This study assesses the interaction effect of political stability and institutional quality on the effective demand of international tourism in 32 African countries during the period 1996-2017. For this purpose, we used the random effects model. In order to consider the endogenous bias, we used the Generalized Moment Method (GMM) in difference estimator. The results reveal that the political stability, the quality of the institutions and their interaction respectively have a positive and significant effect on tourism. These results are robust in different specifications considering the several measure of the political stability and the quality of institutions. Moreover, the results suggest that African countries wishing to develop their tourism sector must seek to improve their stability and the quality of their institutions. Political stability and quality of institutions are necessary for the influx of tourists, but they are not sufficient to make an attractive destination. 
INDEX

Mots-clés : stabilité politique, qualité des institutions, tourisme

Keywords : political stability, institutional quality, tourism

\section{AUTEURS}

\section{TORCIA-CHANELLE BANENGAÏ-KOYAMA}

Économie internationale

Doctorante, Université Felix Houphouët Boigny (Côte d'Ivoire)

banengaichanelle[at]gmail.com

\section{PATRICE ONGONO}

Économie internationale

Docteur, Université de Yaoundé II-Soa (Cameroun)

ongonopatrick[at]yahoo.fr

\section{TRÉSOR EKOM}

Économie des ressources naturelles et de l'environnement

Doctorant, Université Omar Bongo (Gabon)

tresorekom[at]gmail.com 